\title{
Burnout en profesionales de la salud y personal administrativo en una unidad médica de primer nivel
}

\author{
Burnout in health professionals and administrative staff \\ in a medical unit of first level
}

\author{
Sandra Yadira Flores Tesillo y Ana Olivia Ruíz Martínez ${ }^{l}$
}

\begin{abstract}
RESUMEN
La presente investigación aborda la salud mental en una unidad médica de primer nivel, comparando el síndrome de burnout en dos grupos de trabajadores: profesionales de la salud y personal administrativo. Los instrumentos de evaluación empleados fueron un cuestionario de datos sociodemográficos y la Escala de Valoración del Maslach Burnout Inventory Human Services Survey (MBI-HSS). La muestra estuvo conformada por 29 profesionales de la salud (enfermeras y médicos) y 29 trabajadores del área administrativa. Respecto a las variables sociodemográficas, no se encontraron diferencias significativas entre ambos grupos de trabajadores; en cambio, hubo importantes diferencias en la presencia del síndrome en el componente de despersonalización, mayor en el personal administrativo. Se discuten las diferencias entre los puestos, mismas que pueden provocar la despersonalización y no los demás componentes de dicho síndrome.
\end{abstract}

Palabras clave: Burnout; Personal administrativo; Médicos; Enfermeras.

\begin{abstract}
The present study addressed the mental health of health professionals and administrative staff in a first-level medical facility. Measures of burnout were compared between health caregivers (physicians and nurses) and administrative staff of the facility. Measurement included a socio-demographic questionnaire and the Maslach Burnout Inventory Human Services Survey (MBI-HSS) rating scale. A total of 58 members, 29 health professionals and 29 staff workers participated. Results showed significant differences in the depersonalization component of burnout, with the highest scores occurring in the administrative staff. No significant differences between the two groups were found in terms of socio-demographic variables. Differences between the duties that could cause the presence of depersonalization and the lack of other components of the burnout syndrome are discussed.
\end{abstract}

Key words: Burnout; Administrative personal; Doctors; Nurses.

\section{INTRODUCCIÓN}

$\mathrm{L}$

as instituciones de salud requieren trabajadores eficientes y comprometidos con su vocación y con la calidad del servicio, por lo que la propia institución requiere brindar a quienes laboran en ellas un desarrollo de mayor alcance y de duración más extensa que la simple formación escolarizada recibida por los mismos. El desarrollo profesional debe ser una opción empresarial estratégica si la institución u or-

\footnotetext{
${ }^{1}$ Centro Universitario uAEm Zumpango, Universidad Autónoma del Estado de México, Camino Viejo a Jilotzingo, continuación Calle Rayón, Valle Hermoso, 55600 Zumpango, Edo. de México, México, tels.: (591)917-41-40 y (591)917-27-02, correo electrónico: flores_tesillo@hotmail.com. Artículo recibido el 7 de diciembre de 2016 y aceptado el 24 mayo de 2017.
} 
ganización aspira a sobrevivir en un entorno global cada vez más competitivo (Fernández, 2002). El trabajo es indispensable para una vida saludable, tanto por la retribución económica como por el progreso social que implica la realización de la persona y de la comunidad (Nieto, 2000).

Es obvio que un trabajador comprometido es más productivo y útil que uno apático, deprimido, estresado y cansado, de modo que la adecuada salud del trabajador contribuye al crecimiento de la empresa o institución, lo que a la postre trae consigo la mejor la calidad de vida de los trabajadores y sus familias, el bienestar individual y social y la prosperidad económica en el país (Organización Mundial de la Salud [OMS], 2010). La salud mental no únicamente se refiere a la ausencia de trastornos mentales o de enfermedades, sino a la práctica de las potencialidades y capacidades en la vida de las personas, a su interacción social y con el medio ambiente, inherentes a la naturaleza del ser humano y que a su vez condicionan su bienestar individual y colectivo (Secretaría de Salud, 2002).

Sin embargo, el trabajo también puede afectar la salud del trabajador, específicamente la mental, al producir algunas afectaciones, como estrés emocional crónico; agotamiento mental; pérdida progresiva del idealismo y la energía; falta de deseo en alcanzar objetivos; comportamientos indiferentes, distantes e incluso lesivos, y sentimientos de culpa (Domínguez et al., 2012). Tales afectaciones pueden ser generalizadas debido a las condiciones y medio ambiente del trabajo.

En el trabajo, el ser humano siempre ha estado expuesto a riesgos físicos, químicos, ergonómicos y psicosociales que son inherentes al mismo, entre los cuales estos últimos han ido adquiriendo una importancia mundial cada vez mayor (García y Giraldo, 2013).

Dentro de los primeros se encuentran las condiciones de seguridad (instalaciones, maquinaria y herramientas), las características estructurales del lugar de trabajo, los riesgos ambientales, el material escaso y la falta de seguridad (Nieto, 2000), $\mathrm{y}$ entre los factores psicosociales, los horarios inflexibles; la mala comunicación con los compañeros de trabajo, la remuneración insuficiente, el trabajo continuo en horarios extensos, la carga física o mental que implica el trabajo, las demandas laborales excesivas y otros (Carmo et al., 2010). $\mathrm{Si}$ a todos los factores mencionados de origen externo (producidos por la institución) se les agregan las demandas de excelencia profesional (como empatía, comprensión y dedicación), el resultado es una elevada exigencia personal (Greig y Fuentes, 2009) que genera agotamiento y conflicto con los valores personales que paulatinamente conducen a la desilusión, la frustración, la desmotivación $\mathrm{y}$, como consecuencia, al desencadenamiento del proceso del llamado síndrome de burnout (Loaiza, 2010).

El estrés es una respuesta automática ante cualquier situación que se perciba como una demanda o amenaza y que exige de un rápido cambio, en donde lo más importante no es lo que sucede sino la forma en cómo se percibe (Naranjo, 2009).

El estrés y el burnout presentan matices diferentes, por lo que no se debe confundirlos; el estrés (eustrés) puede incluso aumentar el rendimiento de una persona, ayudarla a enfrentar y resolver de forma adecuada las situaciones de la vida diaria y a desarrollar su máximo potencial, mientras que el burnout siempre tendrá consecuencias negativas (Manzano, 2001); además, el primero hace referencia a procesos breves, en tanto que el burnout es considerado como el resultado de una situación prolongada de desgaste (Arias y Zegarra, 2013).

El burnout va más allá de la actitud, pues afecta la conducta y la condición orgánica, y surge como una respuesta al estrés laboral crónico que padece el trabajador y que involucra un estado de agotamiento físico, emocional y cognitivo (Rodríguez y Rivas, 2011).

Maslach y Jackson (1981) propusieron una clasificación de tres dimensiones o componentes del síndrome de burnout: a) despersonalización (DP), que se caracteriza por actitudes negativas, irritabilidad, pérdida de motivación, falta de empatía e incompetencia, insensibilidad, antipatía, etc.; b) cansancio emocional (CE), que se define por una pérdida progresiva de energía, desgaste, agotamiento, fatiga física o psicológica, y c) falta de realización personal en el trabajo (RP), que se describe como respuestas negativas, falta de interés, desmotivación, disminución de la autoestima, inseguridad para alcanzar metas u objetivos, o simplemente su anulación. 
En 2008, Graue, Álvarez y Sánchez sugirieron una forma diferente de caracterizar al síndrome de burnout: a) agotamiento emocional, fatiga y depresión; b) relación de los síntomas con la actividad laboral; c) predominancia de estos síntomas en los ámbitos mentales y conductuales sobre el cansancio físico; d) aparición de los síntomas en personas normales sin antecedentes "psicopatológicos", y e) ineficiencia y pobre desempeño en el trabajo.

Desde una perspectiva psicosocial, el burnout se concibe como un proceso en el que se concentran diversos factores personales y contextuales que lo convierten en uno de los problemas más complejos de la psicología laboral en la actualidad (Aguirre, 2012). Aparece con mayor frecuencia en los trabajadores implicados en interacciones humanas de carácter intenso y duradero (Vilá et al., 2015) y en la esfera de la prestación de servicios con cargas excesivas de trabajo, como los médicos, enfermeras, psicólogos, nutriólogos, psiquiatras y otros profesionales (Graue et al., 2008). Específicamente en el área médica, los profesionales de la salud se ven sometidos en una constante tensión emocional debido a la batalla por recuperar la homeostasis del paciente (Torres y Guarino, 2013). En dicha área, estudios como el de Galván et al. (2014) señalan que los médicos con menor antigüedad tienen una mayor tendencia a padecer burnout; sin embargo, hay que considerar que tal resultado puede depender de la capacidad de adaptación que tienen los médicos al ámbito laboral, y en buena medida a la capacidad de la institución para afrontar sus propios conflictos internos.

Los trabajadores que manifiestan el síndrome de burnout durante mucho tiempo pueden sufrir numerosos problemas de salud física y mental, así como un desequilibrio en las áreas laboral, familiar, social, económica y otras (Torres y Guarino, 2013), además de un deterioro en la calidad de servicios que proporcionan, ya que el síndrome de burnout perturba la eficacia personal (Seguel y Valenzuela, 2014). Así, estos trabajadores pueden llegar al extremo de limitar el sentido de su vida y en muchas ocasiones aislarse socialmente por la incomprensión de su propio estado. Si bien los síntomas iniciales pueden ser leves durante la instalación de dicho síndrome, es necesario que los trabajadores puedan identificar plenamente los sín- tomas y las áreas que se puedan ver afectadas para poder llevar a cabo una intervención adecuada.

Roth y Pinto (2010) señalan que las revisiones clínicas del síndrome de burnout lo muestran como una categoría clínica determinada por una diversidad de síntomas asociados a la depresión, el estrés agudo, la ansiedad y ciertos trastornos de personalidad.

Según Jiménez, González y Garrosa (2001), una vez que se instala el síndrome de burnout en el personal, este puede manifestar síntomas en diferentes áreas personales: cognitivos (pérdida de valores, falta de expectativas, modificación del autoconcepto, distracción, pérdida de la creatividad), conductuales (ausentismo, aumento de la conducta violenta, consumo excesivo de café, alcohol y fármacos, etc.), comportamentales (suicidio, sentimientos de fracaso, falta de competencia personal, carencia de expectativas y horizontes laborales, insatisfacción generalizada, impuntualidad), sociales (evitación de contacto, conflictos interpersonales, aislamiento, evitación profesional) y emocionales; así como impaciencia, deseo de desertar el trabajo, dudas acerca de su propia competencia profesional, baja autoestima, negación de emociones, sentimientos de autocrítica excesiva unidos a una sensación de impotencia, lo que lleva a los trabajadores a la negatividad e irritabilidad) (cfr. Juárez, Haros, Avalos, Guerra y Chacón, 202; Leka, Griffiths y Cox, 2004).

Un factor importante que se debe mencionar dentro de las afectaciones del síndrome de burnout son las manifestaciones actitudinales ante los pacientes que se deterioran. Como consecuencia este tipo de relaciones, los trabajadores (médicos o enfermeras) pueden reducir la eficacia de su intervención. Las principales actitudes que se manifiestan son desconfianza, apatía, cinismo, ironía hacia los pacientes, familiares y compañeros, hostilidad, escasas verbalizaciones en las interacciones sociales, indiferencia, etc. (Lacasta, Aguirán, Fernández y González, 2008).

Rodríguez (2010) subraya que los estudios hechos en Latinoamérica reportan índices bajos de prevalencia de burnout. Entre los estudios llevados a cabo en México, Rodríguez, Oviedo, Vargas, Hernández y Pérez (2009) reportaron una prevalencia de síndrome de burnout en el Hospital Ecatepec de $27 \%$, y de $24 \%$ en el Hospital de Cuautitlán. El es- 
tudio de Carrillo, Gómez y Espinoza (2012) mostró una prevalencia de 30 a 69\% en médicos generales y especialistas, y Pereda, Márquez, Hoyos y Yánez (2009) encontraron 19.6\% de síndrome de burnout en médicos.

Considerando lo anterior, el presente estudio tuvo la finalidad de comparar el síndrome de burnout en dos grupos de trabajadores: personal de salud (enfermeras y médicos) y personal administrativo de una unidad médica de primer nivel, ubicada en la localidad de Zumpango, Edo. de México (México). Es importante señalar que este estudio comparativo con diseño no experimental entre dos grupos de trabajadores tuvo como propósito ofrecer información sobre un sector de trabajadores que no ha sido suficientemente estudiado: el personal administrativo; de igual manera que los médicos, tienen contacto directo y atienden a un gran número de pacientes por día y en muchas ocasiones las relaciones con ellos son conflictivas por las implicaciones derivadas de su trabajo.

\section{MÉTODO}

\section{Participantes}

La muestra seleccionada estuvo constituida por un total de 58 participantes distribuidos en dos grupos. El primero de ellos, referido como grupo "A", se compuso de los 29 profesionales de salud (enfermeras de medicina preventiva y médicos familiares); el segundo grupo, denotado como grupo "B" se constituyó por 29 participantes del personal administrativo. Ambos grupos eran trabajadores de la referida unidad médica de primer nivel. Todos los participantes fueron voluntarios.

\section{Instrumentos}

Se utilizaron dos instrumentos que se aplicaron individualmente a los participantes:

Cuestionario de datos sociodemográficos, que permitió la recolección de datos de variables sociodemográficas como sexo, edad, estado civil, número de hijos, horas de trabajo continuo, número de empleos, antigüedad, escolaridad, tiempo de egreso y distancia de su hogar al trabajo medida en minutos de trayecto.

Escala de Valoración de Maslach Burnout Inventory Human Services Survey (MBI-HSS) (Maslach y Jackson, 1981), que consta de 22 ítems distribuidos en tres escalas: Cansancio emocional (CE), con nueve ítems; Despersonalización (DP) con cinco, y Realización personal (RP) con ocho. Para obtener los resultados, se suman los ítems de acuerdo a la dimensión. Las dimensiones de CE y DP se suman directamente, mientras que en RP se debe invertir la calificación para que se obtenga la "falta de realización personal".

El instrumento presenta las siguientes categorías: bajo, medio o moderado y alto en cada una de las dimensiones. A CE corresponde el siguiente puntaje: bajo: $<16$, moderado: $17-26$, y alto $>27$; a DP: bajo: $<6$, moderado: $7-12$, y alto: $>13$, y a RP: bajo: $>39$, moderado: 38-32 y alto $<31$ (Guevara, Henao y Herrera, 2002). En cuanto a la consistencia interna o fiabilidad. Juárez, Idrovo, Camacho y Placencia (2014) señalaron en su investigación que en los siete estudios hechos en México que especificaron el valor promedio ponderado, este fue de 0.86 en CE, 0.60 en DP y 0.75 en RP. A su vez, Pando, Aranda y López (2015) obtuvieron las siguientes fiabilidades: 0.86 en CE, 0.75 en RP y 0.70 en DP. En la presente investigación, las fiabilidades fueron de $0.83,0.62$ y 0.72 , respectivamente.

\section{Procedimiento}

Previamente a la conducción del estudio, se solicitó la autorización a las autoridades de la institución para aplicar los instrumentos ya mencionados. Luego, a cada participante se le dio a firmar una carta de consentimiento informado y se aplicaron individualmente el cuestionario de datos sociodemográficos y la MBI-HSS. Por último, se agradeció su participación voluntaria a cada uno de los participantes.

\section{Análisis de datos}

El análisis de los datos recabados se llevó a cabo mediante estadística descriptiva: media y desviación estándar; la comparación entre los dos grupos 
de trabajadores se realizó mediante la $t$ de Student de muestras independientes, y con la finalidad de comparar las variables que podrían predecir la presencia de burnout en ambos grupos, se llevó a cabo un análisis de regresión lineal.

\section{RESULTADOS}

Los resultados de esta investigación se desarrollaron de la siguiente manera: se compararon los datos sociodemográficos entre los dos grupos de trabajadores, así como los componentes de la escala de valoración del MBI-HSS en ambos grupos de trabajadores, y finalmente los resultados del análisis de regresión lineal, consistentes en la relación predictiva entre las variables sociodemográficas y los componentes del burnout.

Dentro de la comparación de los datos sociodemográficos, los dos grupos de trabajadores tuvieron una media de edad de 38 años; además, se halló un promedio similar en el número de hijos, antigüedad, número de empleos y demás variables (Tabla 1). Entre ambos grupos no se encontraron diferencias estadísticamente significativas respecto a los mencionados datos, excepto en el grado de escolaridad, en donde los médicos y enfermeras tuvieron un mayor grado de escolaridad (licenciatura, posgrado y especialidad) que los administrativos, siendo esta diferencia estadísticamente significativa $(\mathrm{t}=5.24, p=.000)$.

Tabla 1. Variables sociodemográficas de los grupos participantes.

\begin{tabular}{|c|c|c|c|c|c|c|}
\hline \multirow{2}{*}{$\begin{array}{c}\text { Variables } \\
\text { sociodemográficas }\end{array}$} & \multicolumn{3}{|c|}{ Médicos/enfermeras } & \multicolumn{3}{|c|}{ Personal administrativo } \\
\hline & $\mathbf{n}$ & $\mathbf{M}$ & (DE) & $\mathbf{n}$ & M & (DE) \\
\hline $\begin{array}{l}\text { Hombres } \\
\text { Mujeres }\end{array}$ & $\begin{array}{r}4 \\
25\end{array}$ & $*$ & $\begin{array}{l}* \\
*\end{array}$ & $\begin{array}{r}5 \\
24\end{array}$ & $*$ & $\begin{array}{l}* \\
*\end{array}$ \\
\hline Edad & $*$ & 38.86 & $(10.61)$ & $*$ & 38.93 & (10.63) \\
\hline \multicolumn{7}{|l|}{ Estado civil } \\
\hline $\begin{array}{l}\text { Soltero } \\
\text { Casado } \\
\text { Unión libre } \\
\text { Divorciado }\end{array}$ & $\begin{array}{r}10 \\
16 \\
3 \\
0\end{array}$ & $\begin{array}{l}* \\
* \\
* \\
*\end{array}$ & $\begin{array}{l}\text { * } \\
* \\
* \\
*\end{array}$ & $\begin{array}{r}6 \\
20 \\
2 \\
1\end{array}$ & $\begin{array}{l}* \\
* \\
* \\
*\end{array}$ & $\begin{array}{l}* \\
* \\
* \\
*\end{array}$ \\
\hline Número de hijos & $*$ & 1 & $(1.23)$ & $*$ & 1 & $(0.98)$ \\
\hline Horas de trabajo & $*$ & 8 & $(3.54)$ & $*$ & 7 & $(1.75)$ \\
\hline Número de empleos & $*$ & 1 & $(0.78)$ & $*$ & 1 & $(1.05)$ \\
\hline Antigüedad & $*$ & 12 & $(9.32)$ & $*$ & 12 & $(8.10)$ \\
\hline \multicolumn{7}{|l|}{ Escolaridad } \\
\hline $\begin{array}{l}\text { Secundaria } \\
\text { Preparatoria } \\
\text { Licenciatura } \\
\text { Posgrado } \\
\text { Especialidad }\end{array}$ & $\begin{array}{r}0 \\
0 \\
17 \\
9 \\
3\end{array}$ & $\begin{array}{l}* \\
* \\
* \\
* \\
*\end{array}$ & $\begin{array}{l}* \\
* \\
* \\
* \\
*\end{array}$ & $\begin{array}{r}1 \\
19 \\
8 \\
1 \\
0\end{array}$ & $\begin{array}{l}* \\
* \\
* \\
* \\
*\end{array}$ & $\begin{array}{l}* \\
* \\
* \\
* \\
*\end{array}$ \\
\hline $\begin{array}{l}\text { Tiempo de egreso } \\
\text { de su profesión en años }\end{array}$ & $*$ & 14 & $(16.10)$ & $*$ & 16 & $(10.16)$ \\
\hline $\begin{array}{l}\text { Transporte en minutos } \\
\text { del hogar al trabajo }\end{array}$ & $*$ & 43 & $(27.61)$ & $*$ & 39 & (37.18) \\
\hline
\end{tabular}

En cuanto a la valoración del síndrome de burnout por componentes, la Tabla 2 muestra la calificación que obtuvo la población total en términos de porcentajes respecto a los componentes del MBIHSS en ambos grupos de trabajadores. 
Tabla 2. Calificación obtenida pos la población total (en porcentaje) en los componentes del MBI-HSS en los dos grupos.

\begin{tabular}{|l|c|c|c|c|c|c|}
\hline \multirow{2}{*}{$\begin{array}{c}\text { Nivel } \\
\text { de calificación }\end{array}$} & \multicolumn{3}{|c|}{ Personal administrativo } & \multicolumn{3}{c|}{ Médicos/enfermeras } \\
\cline { 2 - 7 } & \multicolumn{2}{|c|}{ Componentes del MBI-HSS } & \multicolumn{2}{c|}{ Componentes del MBI-HSS } \\
\cline { 2 - 7 } & CE & DP & RP & CE & DP & RP \\
\hline Alto & 10.34 & 20.68 & 20.68 & 10.34 & 3.44 & 6.89 \\
\hline Moderado & 17.24 & 27.58 & 13.79 & 6.89 & 20.68 & 13.79 \\
\hline Bajo & 72.41 & 51.72 & 65.51 & 82.75 & 75.86 & 79.31 \\
\hline
\end{tabular}

Nota: $\mathrm{CE}=$ Cansancio emocional, $\mathrm{DP}=$ Despersonalización, $\mathrm{RP}=$ Realización personal.

Se puede apreciar que la mayoría del personal administrativo y el de salud estudiados se encuentra con niveles bajos de burnout; sin embargo, hubo trabajadores ubicados en niveles altos y medios, sobre todo del personal administrativo.

Respecto a los niveles promedio en que se hallaban ambos tipos de trabajadores, el personal administrativo se ubicó en un nivel bajo en CE y moderado en los componentes DP y RP. Un nivel moderado en cualesquiera de los tres componentes del síndrome de burnout significa que los trabajadores manifiestan algunas afectaciones conductuales, emocionales, sociales o cognitivas en dichos componentes, considerables en cuanto a la disminución de su calidad de vida; a su vez, el personal de médicos y enfermeras se mantuvo en un promedio bajo en las tres dimensiones $(9.79,4.27$ y 40.96, respectivamente).

En el análisis de las comparaciones entre el puesto y los componentes de la escala de valoración del MBI-HSS, los resultados fueron los siguientes: en el componente de CE no hubo diferencias significativas $(\mathrm{t}=-.86, p=.390)$, al igual que en el componente de RP, en el que no se encontraron diferencias estadísticamente significativas $(\mathrm{t}=1.60$, $p=.115)$. No obstante, en el componente de DP se asume que existen en efecto diferencias significativas $(\mathrm{t}=-2.3, p=.025)$, obteniendo una mayor puntuación los trabajadores administrativos, lo que significa que estos últimos, a diferencia del personal de salud, son más susceptibles a manifestar actitudes de frialdad y distanciamiento, sentimientos de impotencia, desafecto y apatía con los pacientes, lo que claramente puede disminuir la calidad del trabajo proporcionado a los mismos, originando con ello afectaciones a otras áreas de la institución de salud.

La regresión lineal sirve como referencia para conocer si las variables sociodemográficas tomadas en cuenta en este estudio ayudan a predecir la presencia de los componentes del burnout en los diferentes grupos de trabajadores ya que, como se pudo observar anteriormente, hubo una diferencia significativa en la categoría de DP a favor de los trabajadores administrativos. Los resultados de la regresión señalan lo siguiente: $a$ ) en el personal de salud se encontraron relaciones significativas de las variables Tiempo de egreso y Distancia hogar-trabajo que predicen en $32.2 \%$ la presencia de DP. b) En el personal administrativo se halló que la escolaridad predice en $20.8 \%$ la DP y en $20.5 \%$ el CE, en tanto que la distancia hogar-trabajo predice en $16.5 \%$ el CE; por último, la distancia al trabajo también afecta en $16.5 \%$ la falta de RP.

Las variables sociodemográficas restantes, como el sexo, estado civil, edad, número de hijos, horas de trabajo y número de empleos, no resultaron predictoras de ninguna de las dimensiones del burnout.

\section{DISCUSIÓN}

Múltiples investigaciones hacen referencia de que el personal de salud (médicos y enfermeras) es más vulnerable a padecer síndrome de burnout, y un ejemplo claro se encuentra en la investigación de Feliciano, Kobacs y Wanick (2005), que refieren que dichos profesionales están por lo regular tan comprometidos a brindar soluciones a los problemas de salud de los pacientes que tienden al agotamiento emocional, depresión y sentimientos de insuficiencia.

Afortunadamente, en el presente estudio predominó un bajo nivel de burnout en ese personal, seguido por los niveles medio y alto. No obstante, si se consideran las indicaciones del instrumento, que señalan que alcanzar el nivel moderado en cualquiera de los componentes implica sufrir afecciones psicológicas que requieren atención, en- 
tonces hasta una quinta parte del personal de salud cumple tal condición, especialmente por las puntuaciones elevadas en la despersonalización. La comparación de estos resultados con otros estudios permite hallar convergencias y divergencias; por ejemplo, respecto al estudio de Aranda, Pando, Torres, Salazar y Franco (2005), hecho en población mexicana, los participantes del presente estudio mostraron menos cansancio y más despersonalización, pero al comparar estos datos con los obtenidos por Galván et al. (2014) con médicos en Argentina, se encuentra menos cansancio y menos despersonalización.

En cuanto a las variables sociodemográficas asociadas al burnout en los profesionales de salud, destacaron el tiempo de egreso de los estudios y la distancia entre el hogar y el trabajo; sin embargo, estos datos no coinciden con los de otros estudios (Aranda et al., 2005; Galván et al., 2014), que han encontrado distintas variables asociadas, tales como ser soltero, estar sobrecapacitado para el puesto, tener mayor antigüedad en la institución y cubrir guardias o jornadas mayores de 36 horas. Lo anterior sugiere que los factores asociadas al burnout pueden variar de acuerdo a los contextos laborales o geográficos, por lo que se requerirían estudios transculturales.

En el caso del personal administrativo de la institución, también se halló una tendencia hacia los niveles bajos de burnout, con variaciones en sus componentes. El cansancio emocional se ubicó en un nivel bajo, mientras que la despersonalización y la falta de realización personal lo hicieron en niveles moderados. Al comparar estos resultados con los datos obtenidos de otros trabajadores administrativos, el porcentaje de burnout en la muestra de estudio osciló entre 10 y $21 \%$, mientras que en el estudio de De los Ríos, Ocampo, Laneros, Ávila y Tristán (2007), hecho en el personal administrativo educativo de San Luis Potosí, rebasó en $50 \%$ los niveles altos, lo que evidencia la necesidad de realizar otros estudios con muestras de diferentes perfiles administrativos que hagan posible dilucidar si los resultados dependen del área de trabajo. En esta investigación, los factores asociados al burnout del personal administrativo fueron la mayor distancia al trabajo y una menor escolaridad, pero en el estudio referido de De los Ríos et al. (2007) tales aspectos fueron distintos: sexo femenino, mayores de 36 años, casadas, con una antigüedad mayor a 15 años e insatisfacción laboral.

En cuanto a la comparación entre los dos tipos de personal con los componentes del inventario utilizado, se encontraron diferencias significativas entre ambos grupos de trabajadores en el componente de despersonalización, donde el personal administrativo alcanzó mayores índices. A este respecto, Llorens, García y Salanova (2005) señalan las consecuencias que ello tiene en el servicio a los usuarios, pues la despersonalización se caracteriza por actitudes negativas, insensibilidad hacia los usuarios del servicio y respuestas de tipo impersonal; en otras palabras, por un endurecimiento afectivo.

Es importante mantener una línea de investigación del burnout en el país, ya que los resultados del personal administrativo de la presente investigación muestran un mayor riesgo de padecerlo, lo que indica que los médicos no son los únicos trabajadores vulnerables ante tal síndrome; de hecho, cualquier trabajador puede sufrir estrés, ante el cual reacciona de diferentes formas para afrontarlo según los recursos de que dispone (Oswaldo, Días, Rueda y Filho, 2012).

El personal de salud y el administrativo tuvieron una percepción diferente acerca de sus actividades laborales. Es importante referir que durante las aplicaciones a los participantes, algunos miembros del primero de estos dos grupos señalaron que el trabajo implicaba para ellos un lugar de distracción a los problemas familiares y que disfrutaban su labor; por el contrario, los comentarios de los del segundo fueron en el sentido de que su trabajo les provocaba tensiones constantes.

Un punto importante en la investigación es que una de las variables sociodemográficas importantes fue la escolaridad, que mostró una influencia significativa en el análisis predictivo, de tal forma que una mayor escolaridad se asocia con una menor despersonalización. Se requiere, pues, de investigaciones más acuciosas que indiquen de qué manera actúa la educación en ese sentido; es posible que la educación y la profesión (enfermería o medicina), tareas exigentes de suyo, pueden formar parte de un plan de vida y generar un mayor disfrute; en cambio, el personal administrativo, con menos preparación y reconocimiento, 
debe mantener metas y objetivos claros para su desarrollo personal y profesional que les hagan posible desempeñarse en áreas laborales según sus habilidades, condiciones físicas, preparación académica, deseos, aptitudes, personalidad y otros factores.

Otras variables sociodemográficas, como la edad, las horas de trabajo o el número de hijos no resultaron ser relevantes. De esta manera el burnout no depende de esas variables, sino de cuestiones personales y del propio carácter del trabajo, como el número de pacientes atendidos, la relación entre el personal y el paciente, el significado laboral, el reconocimiento laboral, etcétera.

Estos resultados no se anticipaban ya que en algunas investigaciones (Aranda, Pando, Velázquez, Acosta y Pérez, 2003; Feliciano et al., 2005; Guevara et al., 2002) hallan que variables sociodemográficas tales como la edad, el sexo, el número de trabajos o las horas de la jornada laboral pueden influir directamente en la vulnerabilidad de los trabajadores para padecer el síndrome de burnout.

En conclusión, en el área de la salud se desempeñan tanto profesionales de salud como personal administrativo, no obstante lo cual el estudio de burnout se ha centrado en los primeros. Por tal motivo, se comparó el burnout en ambos tipos de trabajadores, encontrando que predomina el nivel bajo y moderado y que la diferencia se encuentra en la despersonalización, en la que el personal administrativos muestra más frialdad y distanciamiento y menos empatía hacia los usuarios, lo que puede estar asociado a su menor escolaridad, el mayor tiempo de egreso y la distancia del hogar al trabajo.

Tal comparación muestra la importancia de atender no solo a los médicos y enfermeras, sino también al personal administrativo como un eslabón importante en los servicios de salud, el cual generalmente atiende a una gran cantidad de usuarios y constituye el primer contacto que brinda información y realiza los trámites necesarios para emprender las acciones de intervención sanitaria necesarias en el cuidado de la salud. Dicho personal requiere apoyo para adaptarse a las exigencias de su trabajo, de manera que pueda dirigir y afrontar sus actividades diarias en los ámbitos personal, familiar, organizacional y social.

En consecuencia, se sugiere que en futuras investigaciones se trabaje con muestras representativas del sector salud, abordar los estilos de afrontamiento y emplear diferentes tipos de profesionistas y trabajadores y profundizar en la variable de escolaridad. Asimismo, sería conveniente complementarlas con una aproximación cualitativa que permita indagar cuestiones relacionadas al significado, representaciones y carga afectivo-emocional sobre el trabajo que realiza cada tipo de empleado y los diferentes tipos de trato entre ellos y los pacientes.

\section{REFERENCIAS}

Aguirre, J. (2012). Organización, expectativas y salud mental. Un análisis psicosocial en el contexto universitario. Revista Iberoamericana para la Investigación y el Desarrollo Educativo, 24, 37.

Aranda, C., Pando, M., Torres, T., Salazar, J. y Franco, S. (2005). Factores psicosociales y síndrome de burnout en médicos de familia. Anales de la Facultad de Medicina, 66(3), 1-9.

Aranda, C., Pando, M., Velázquez, I., Acosta, M. y Pérez., M. (2003). Síndrome de burnout y factores. Revista de Psiquiatría de la Facultad de Medicina de Barcelona, 30(4), 193-199.

Arias, W.L. y Zegarra, J. (2013). Clima organizacional, síndrome de burnout y afrontamiento en trabajadores de un hospital oncológico de Arequipa. Revista de Psicología (Trujillo), 15(1), 37-54.

Carmo, M., Chaves, M., Barcellos, R., Almeida, L., Oliveira, I., y Pedrao, L. (2010). Exceso de trabajo y agravios mentales a los trabajadores de la salud. Revista Cubana de Enfermería, 26(1), 52-64.

Carrillo, R., Gómez, K. y Espinoza, I. (2012). Síndrome de burnout en la práctica médica. Medicina Interna de México, 2(28), $578-584$.

De los Ríos C., J.L., Ocampo M., M., Landeros L., M., Ávila R., T.L. y Tristán A., L.A. (2007). Burnout en personal administrativo de una dependencia gubernamental: Análisis de sus factores relacionados. Investigación y Ciencia, 37, 26-34.

Domínguez, J., Herrera, F., Villaverde, M., Padilla, I., Martínez, M. y Domínguez, J. (2012). Síndrome de desgaste profesional en trabajadores de atención a la salud en el área sanitaria de Ceuta. Atención Primaria, 44(1) 30-35. 
Feliciano, K., Kobacs, M. y Wanick S. (2005). Los sentimientos de los profesionales del servicio de emergencias pediátricas: reflexivos sobre burnout. Pernambuco (Brasil): Universidad de Pernambuco.

Fernández, N. (2002). El desarrollo profesional de los trabajadores como ventaja competitiva de las empresas. Cuadernos de Gestión, 2(1), 65-90.

Galván M., E., Vassallo J., C., Rodríguez, S., Otero, P., Montonati, M.M., Cardigni, G., Buamscha, D.G., Rufach, D., Santos, S., Moreno, R.P. y Sarli, M. (2014). Síndrome de desgaste profesional (burnout) en médicos de unidades de cuidados intensivos pediátricos en la Argentina. Archivos Argentinos de Pediatría, 53(1), 29-36.

García, C. y Giraldo, J. (2013). Burnout en personal de salud. Tesis de doctorado. Medellín (Colombia): Universidad CES. Disponible en línea: http/bdigital.ces.edu.co:8080/repositorio/bitstream/10946/1842/2/Burnout_personal_de_salud.pdf.

Graue, E., Álvarez, R. y Sánchez, M. (2008). El síndrome de burnout: la despersonalización, el agotamiento emocional y la insatisfacción en el trabajo como problemas en el ejercicio de la medicina y el desarrollo profesional. México: Editores de Textos Mexicanos, S.A. de C.V.

Greig, D. y Fuentes, X. (2009). Burnout o agotamiento en la práctica diaria de la medicina. Revista Chilena de Cardiología, 28(4), 403-407.

Guevara, C., Henao, D. y Herrera, J. (2002). Síndrome de desgaste profesional en médicos internos y residentes. Colombia Médica, 35(4), 173-178.

Jiménez, B., González, J. y Garrosa, E. (2001). Desgaste profesional (burnout), personalidad y salud percibida. En J. Buendía y F. Ramos (Eds.): Empleo, estrés y salud (pp. 59-83). Madrid: Pirámide.

Juárez, A., Idrovo, A., Camacho, A. y Placencia, O. (2014). Síndrome de burnout en población mexicana: Una revisión sistemática. Salud Mental, 37, 159-176.

Juárez, M., Haros, Y., Avalos, J., Guerra, R. y Chacón, S. (2012). Factores de riesgo relacionados con el síndrome de burnout en el personal de enfermería de un hospital de Ciudad Juárez, Chihuahua, México. Cultura Científica y Tecnológica, 57, 289-296.

Lacasta, M., Aguirán, P., Fernández, E. y González, M. (2008). Consecuencias del síndrome del burnout. En M. González, M. Lacasta y A. Ordoñez (Eds.): El síndrome de agotamiento profesional en oncología (pp. 35-42). Madrid: Editorial Médica Panamericana.

Leka, S., Griffiths, A. y Cox, T. (2004). Work organisation and stress. Systematic problem approaches for employers, managers and trade union representative. Nottingham, UK: University of Nottingham.

Llorens, S., García, M. y Salanova, M. (2005). Burnout como consecuencia de una crisis de eficacia: un estudio longitudinal en profesores de secundaria. Revista de Psicología del Trabajo y Organizaciones, 21(1-2), 55-70.

Loaiza, E. (2010). Las organizaciones tóxicas: consecuencia fatal de una organización exitosa que pierde su camino. UNACIENCIA, Revista de Estudios e Investigaciones, 4, 1-12.

Manzano, G. (2001). Estrés crónico laboral asistencial (burnout) en las administraciones públicas. Dirección y organización. Revista de Ingeniería de Organización, 15, 1-12.

Maslach, C. y Jackson, S. (1981). The measurement of experienced burnout. Journal of Occupational Behaviour, 2, 99-113.

Naranjo, M. (2009). Una revisión teórica sobre el estrés y algunos aspectos relevantes de éste en el ámbito educativo. Revista de Educación, 33(2), 171-190.

Nieto, H. (2000). Salud laboral: la salud de los trabajadores de la salud. Buenos Aires: Universidad de Buenos Aires. Disponible en línea: www.fmed.uba.ar/depto/sal_seg/la_salud_de_los_trabajadores_de_la_salud.pdf.

Organización Mundial de la Salud (2010). Entornos laborales saludables: fundamentos y modelos de la OMS. Contextualización, prácticas y literaturas de apoyo. Disponible en línea: http://www.who.int/occupational_health/evelyn_hwp_spanish.pdf.

Oswaldo, Y., Días, E., Rueda, V. y Filho, O. (2012). Impacto de los estresores laborales en los profesionales y en las organizaciones análisis de investigaciones publicadas. Invenio, 29(15), 67-80. Disponible en línea: http://www.redalyc.org/articulo. oa? id $=87724146007$.

Pando, M., Aranda, C. y López, M. (2015). Validez factorial del Maslach Burnout Inventory-General Survey en ocho países latinoamericanos. Ciencia y Trabajo, 52, 28-31.

Pereda, L., Márquez, F., Hoyos, M. y Yánez, M. (2009). Síndrome de burnout en médicos y personal paramédico. Salud Mental, 32(5), 1-6.

Rodríguez, C., Oviedo, A., Vargas, M., Hernández, V. y Pérez, M. (2009). Prevalencia del síndrome de burnout en el personal de enfermería de dos hospitales del Estado de México. Fundamentos en Humanidades, 19, 179-193.

Rodríguez, M. (2010). Burnout: un fenómeno creciente. Psicología desde el Caribe, 26, 17-20. Disponible en línea: http://www. redalyc.org/articulo.oa?id $=21319039001$.

Rodríguez, R. y Rivas, S. (2011). Los procesos de estrés laboral y desgaste profesional (burnout): diferenciación, actualización y líneas de intervención. Medicina y Seguridad del Trabajo, 57(1), 1-17. 
Roth, E. y Pinto, B. (2010). Síndrome de burnout, personalidad y satisfacción laboral en enfermeras de la ciudad de La Paz. Ajayu, $8(2), 62-100$.

Secretaría de Salud (2002). Programa de Acción en Salud Mental. México: SS. Disponible en línea: www.salud.gob.mx/unida$\mathrm{des} /$ cdi/documentos/pasm_intro.pdf.

Seguel, F. y Valenzuela, S. (2014). Relación entre la fatiga laboral y el síndrome de burnout en personal de enfermería de centros hospitalarios. Enfermería Universitaria, 11(4), 119-127.

Torres, B. y Guarino, L. (2013). Diferencias individuales y burnout en médicos oncólogos venezolanos. Universitas Psychologica, 12(1), 95-104.

Vilá, M., Cruzate, C., Orfila, F., Creixell, J., González, M. y Davins, J. (2015). Burnout y trabajo en equipo en los profesionales de atención primaria. Atención Primaria, 45(1), 14-26. 